\title{
Experimental Performance Evaluation of Multihop IEEE 802.15.4/4g/4e Smart Utility Networks in Outdoor Environment
}

\author{
Chin-Sean Sum, Ming-Tuo Zhou, Fumihide Kojima, and Hiroshi Harada \\ National Institute of Information and Communications Technology (NICT), Tokyo, Japan \\ Correspondence should be addressed to Chin-Sean Sum; cssumresearch@gmail.com
}

Received 29 September 2016; Accepted 4 January 2017; Published 22 March 2017

Academic Editor: Patrick Seeling

Copyright ( 2017 Chin-Sean Sum et al. This is an open access article distributed under the Creative Commons Attribution License, which permits unrestricted use, distribution, and reproduction in any medium, provided the original work is properly cited.

\begin{abstract}
This paper presents the experimental performance evaluation results of the IEEE 802.15.4/4g/4e Smart Utility Networks (SUN) in applications suited for outdoor environment. SUN is an advanced wireless communications network designed for reliable, low data rate, and low energy consumption networks for command-and-control applications like utility service, sensor network, and so on. IEEE $802.15 .4 \mathrm{~g} / 4 \mathrm{e}$ is the international standard for SUN supported by multiple utility providers and product vendors. In this paper, a comprehensive field test was conducted by employing the implementation we have developed to evaluate the performance of the SUN devices based on IEEE $802.15 .4 / 4 \mathrm{~g} / 4 \mathrm{e}$ standard. The output power of the implementation is $250 \mathrm{~mW}$ for extended range, reducible to $20 \mathrm{~mW}$ for short-range scalability and battery preservation. Results showed that in an outdoor line-of-sight environment, the achievable one-hop range of a $50 \mathrm{kbps}$ SUN device was $450 \mathrm{~m}$. Next, in a non-line-of-sight environment involving typical residential concrete building, the communications could be established penetrating obstructions to reach above the 11th storey, reaching the performance degradation limits at the 20th storey. Next, the network of the SUN system was proven to be capable of supporting a typical multihop tree network in a dense populated building, meeting the required performance by the standard.
\end{abstract}

\section{Introduction}

Low-data-rate and low-energy-consumption wireless communication systems is one of the essential technologies supporting applications such as smart grid/utility networks [1, 2], machine-to-machine communications [3], and advanced home/sensor networks [4]. This genre of applications outlines a unique set of system requirements different from communication systems designed specifically for high speed applications. The requirements are not limited but in correspondence particularly to features such as enhanced network robustness/reliability, extended operating range, prolonged battery lifespan, and improved cybersecurity. The wireless community has been diligently working to standardize the communication protocols to enable a globally compatible system for such applications. A usage model of such application type is given in Section 3.

IEEE 802.15.4 [5] is an international standard specifying communication protocols for Low Rate Wireless Personal Area Network (LR-WPAN). This base standard is later amended by IEEE $802.15 .4 \mathrm{~g}$ [6] and IEEE $802.15 .4 \mathrm{e}$ [7], with additional enhancement in industrial application features and radio communications mechanism suited for SUN. In these standards, the wireless community has included various functionality to equip the LR-WPAN communication system, with advanced features such as robust multihop, power saving, interference detection/avoidance, and optimized physical layer design. The overview of physical (PHY) and medium access control (MAC) layer design in general and those contributed by the authors are detailed in Section 4 .

Following system design and extensive simulations [2] of the IEEE $802.15 .4 / 4 \mathrm{~g} / 4 \mathrm{e}$ system, the authors have also included the efforts to build a prototype based on the specification. The SUN device is operational in the sub-1-GHz band, with output power of $20 \mathrm{~mW}$, expandable to $250 \mathrm{~mW}$. Supported data rates are in the order of several hundreds of kbps, meeting the typical requirements for commandand-control applications. The description of the prototype building is given in [8]. Section 5 provides an overview of the prototype implementation. 
By using the developed prototype, field trials were conducted to evaluate the performance of the SUN system. The tests were conducted in Singapore, covering multiple scenarios that represent the typical environment most wireless communication networks may encounter. These scenarios involve open air line-of-sight (LOS) achievable range, outdoor nonline-of-sight (NLOS) achievable range, dense urban area multihop networks establishment, and range extension for suburban networks. The test results of the field trial were gathered and analyzed to determine if the SUN system is capable of meeting the requirements set forth by the use case scenarios in Section 3. The test deployment scenarios and network performance evaluation are described in Sections 6 and 7 , respectively. Finally, detailed discussions relating the use case scenarios and the evaluation results are presented in Section 8.

One of the main challenges in this project is to enable the node-to-node communications to overcome different buildings and geographical terrains. As shown in [1], the SUN is primarily a field area network operating in outdoor scenarios, connecting the wide area network to the premises. In outdoor scenarios, there are bound to be natural and manmade obstacles which may compromise the effectiveness of the network. In these scenarios, a multihop system operating in the sub- $\mathrm{GHz}$ band is very efficient in increasing the efficiency and robustness of the network.

To the best knowledge of the authors, the evaluation of IEEE 802 standards SUN system, based on simulation and theoretical means, is minimal, let alone evaluation based on realistic field trials. Hence, the contribution of this paper is threefold: (a) provided system design and prototype implementation for SUN device; (b) conducted field tests for multiple outdoor test case scenarios; (c) verified the test results relating to practical SUN deployment in the real world. It is our strong belief that this paper should provide a viable reference for both the research and industrial community.

\section{Related Works}

This section lists the related studies on system design, performance evaluation, and interference management in the SUN area. Documents in $[1,9]$ show the interrelated domains of smart grid/utility and outlining the communications entity in the field area network for SUN. In $[2,10]$, we have recorded extensive and detailed performance evaluation on both the IEEE 802.15.4g frequency shift keying (FSK) and orthogonal frequency division multiplexing (OFDM) PHY layers. Optimization and enhancement on system design were studied in [11-14]. In [11], energy efficient mechanism for SUN was explored to prolong battery lifespan, while, in [12, 13], low duty systems and beaconless synchronization methods were investigated for the same reason. Effectiveness of a wireless mesh network is discussed in $[15,16]$. Besides system design, we have also conducted numerous studies on the impact of interference. In [17], the achievable communication range and tolerable interference range were investigated. In [18], the impact of homogeneous and heterogeneous interferer on SUN systems was evaluated. Then in $[19,20]$, we have proposed methods to manage and mitigate the interference from different sources. To further our understanding of the SUN system behavior, besides theoretical and simulation works mentioned, we have diversified our studies into field trials with actual implementation, as recorded in this paper. Besides our field trial, there was also another implementation recorded in [21-23].

\section{Smart Utility Networks Usage Model}

SUN is the next-generation utility network equipped with communication technologies facilitating enhancements to optimize the consumption of resources. Examples of applications are Advanced Metering Infrastructure (AMI), Demand Response (DR), Distribution Automation (DA), and others, developed to enable efficient resource management [24, 25]. Targeted utility services for these applications are typical, but not limited to power, water, natural gas, and sewage utility systems. By enabling two-way communications between the service provider and end-user terminals in these utility networks, advanced command-and-control features can be applied to optimize the resources while maintaining minimum cost, ultimately benefiting both utility providers and utility consumers.

A typical usage model of the Smart Utility Network (SUN) is illustrated in Figure 1. On the utility consumers' end, utility meters are equipped with communication modules that form a wireless network. The network is commonly deployed for meters and other sensors in residential areas, office, and industrial sites. In these areas, the network should be sufficient to provide coverage to different scenarios of building structure and distribution. In multistorey buildings for residential and office purposes, the network is expected to overcome the attenuation due to the walls in between nodes. At the ground level, the network should also be able to cover the distance across nodes in both line-ofsight (LOS) and non-line-of-sight (NLOS) environments. For nodes located at extended distances, multihop operations can be employed to provide additional coverage, particularly into suburban regions where the population is relatively sparse. The aforementioned network is the SUN network that facilitates communication among several thousands of nodes to exchange information. The SUN network is then extended all the way to the utility collection/control station via wide area network (WAN). The WAN may be wired or connected through wireless channel, depending on different system demands.

In order to provide the service described, SUN should possess capabilities to support the following use cases:

(i) Use case 1: provide coverage that supports the typical distance among nodes deployed in LOS facilities on the ground.

(ii) Use case 2: provide coverage that overcomes penetration of obstructions among NLOS entities in multiple-storey buildings.

(iii) Use case 3: form a network that covers the field or neighborhood area networking system connecting multiple nodes in a dense environment. 


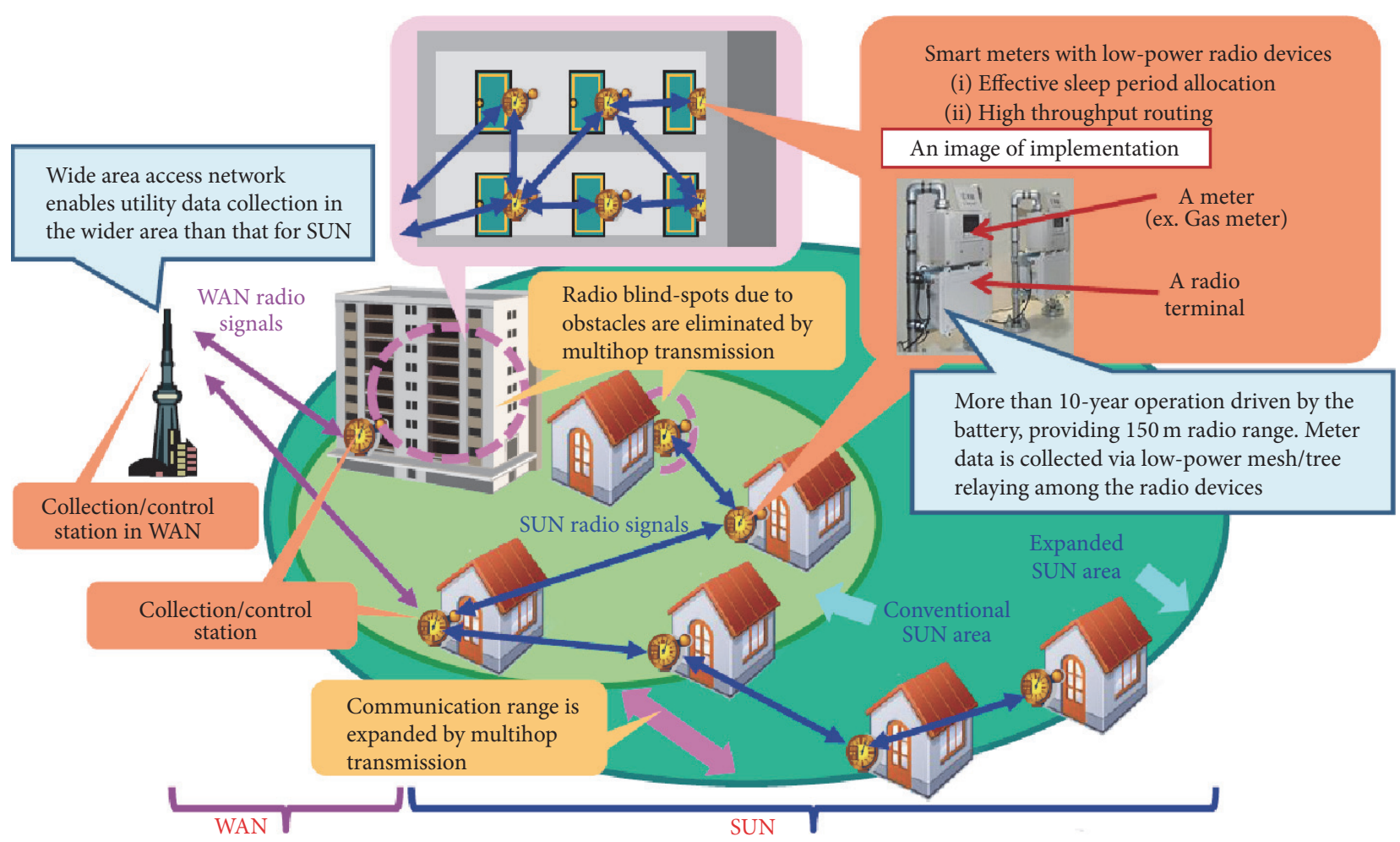

FIGURE 1: Illustration of typical usage model for Smart Utility Networks.

(iv) Use case 4: form an extended network that expands the effective range to multiples of order of a one-hop signal into areas with sparse population.

These four use cases can be combined and derive a generic usage model for an outdoor networking system in an urban area with primarily buildings and roads. This matches well with the deployment scenario in a typical urban state, such as Singapore.

\section{IEEE 802.15.4g and IEEE 802.15.4e}

IEEE 802.15.4g [6] and IEEE 802.15.4e [7] are amendments of IEEE 802.15.4-2011 [5], respectively, dealing with physical layer and MAC layer requirements for applications such as SUN. IEEE 802.15.4g targeted at usage scenario of neighborhood area network (NAN), where utility meters are deployed outdoor and form mesh/ad hoc networks. Comparing with the baseline standard, such usage scenarios present more technical challenges due to harsher environment.

IEEE $802.15 .4 \mathrm{e}$ intends to support a wide range of industrial and commercial applications that require lower latency, higher robustness, and deterministic behaviors. To achieve this, $802.15 .4 \mathrm{e}$ has specified a number of mechanisms targeting different application domains with different features [7]. On the other hand, IEEE 802.15.4e has also developed additional MAC functions to enhance the general capabilities, like low energy (LE), information element (IE), enhanced beacons (EB) and enhanced beacon requests (EBR), and so forth. This section introduces selected 802.15.4e MAC features that has been implemented in the test-bed devices.

4.1. Regulation. The field trial was carried out in Singapore so the developed SUN devices must comply with the local shortrange device regulation which is in [26]. Three possible frequency bands in Singapore are allocated for applications like smart metering, that is, $866-869 \mathrm{MHz}, 920-925 \mathrm{MHz}$, and 2.4000-2.4835 GHz. The maximum allowed output powers in the three bands are $10 \mathrm{~mW}$ ERP (Effective Radiated Power), $500 \mathrm{~mW}$ ERP, and $100 \mathrm{~mW}$ EIRP (Equivalent Isotropic Radiated Power), respectively.

4.2. PHY Layer. There are three different physical layers (PHYs) specified in the $802.15 .4 \mathrm{~g}$ standard, that is, multirate and multiregional FSK (MR-FSK) PHY, MR-OQPSK PHY, and MR-OFDM PHY. MR-FSK is mandatory and the other two are optional. The different $802.15 .4 \mathrm{~g}$ PHYs can operate in the same location and within the same frequency band. This subsection introduces MR-FSK PHY as it is mandatory and implemented by the test-bed device of this study.

4.2.1. Advantages. Adopting MR-FSK offers two advantages: (1) it provides good transmit power efficiency due to the constant envelope of the transmit signal; (2) most of the deployed systems in US are based on FSK modulation schemes, including ones operating in the $920-928 \mathrm{MHz}$ license-exempt band and the sub-GHz licensed band. 
TABLE 1: Frequency bands and data rates of MR-FSK PHY in different countries.

\begin{tabular}{lccc}
\hline Number & Frequency band $(\mathrm{MHz})$ & Regulatory domain & Data rates $(\mathrm{kbps})$ \\
\hline 1 & $169.400-169.475$ & Europe & $2.4 ; 4.8 ; 9.6$ \\
2 & $450-470$ & US & $5.8 ; 9.6$ \\
3 & $470-510$ & China & $50 ; 100 ; 200$ \\
4 & $779-787$ & China & $50 ; 100 ; 200$ \\
5 & $863-870$ & Europe & $10 ; 20 ; 40$ \\
6 & $896-901$ & US & $10 ; 20 ; 40$ \\
7 & $901-902$ & US & $50 ; 150 ; 200$ \\
8 & $902-928$ & US & $50 ; 150 ; 200$ \\
9 & $917-923.5$ & Korea & $50 ; 100 ; 200 ; 400$ \\
10 & $920-928$ & Japan & $10 ; 20 ; 40$ \\
11 & $928-960$ & US & $50 ; 100 ; 200 ; 400$ \\
12 & $950-958$ & Japan & $10 ; 20 ; 40$ \\
13 & $1427-1518$ & US, Canada & $50 ; 150 ; 200$ \\
14 & $2400-2483.5$ & Worldwide &
\end{tabular}

4.2.2. Operation Frequency Bands. IEEE 802.15.4g applications are supported by multiple regulatory-domain-specific frequency band allocation. A total of 14 frequency bands listed in Table 1 are identified for the MR-FSK PHY based on the short-range-device (SRD) regulations of different countries. Although Singapore was not included in the table, the SRD frequency band of 920-925 MHz falls in Japan and US bands of 920-928 MHz, and the band 866-869 MHz falls in Europe band $860-870 \mathrm{MHz}$.

Additionally, the spectrum mask of the data rate modes can be found in [6].

4.2.3. Data Rates. The MR-FSK PHY supports multiple data rates for different frequency bands/countries as listed in Table 1, where the data rates with star marker are mandatory and others are optional. According to the SRD operating frequency band (920-925 MHz) in Singapore, the supported MR-FSK data rates are $50 \mathrm{kbps}, 100 \mathrm{kbps}, 150 \mathrm{kbps}, 200 \mathrm{kbps}$, and $400 \mathrm{kbps}$ in Singapore.

4.2.4. PPDU Format. MR-FSK PHY has two PPDU formats as shown in Figure 2, one for MR-FSK without mode switch, the other for MR-FSK with mode switch. Mode switch is a mechanism by which MR-FSK PHY changes symbol rate and/or modulation scheme on a packet-by-packet basis. The PPDU format of MR-FSK PHY without mode switch is mandatory while the other is optional.

The first part of a PPDU is synchronization header (SHR) which includes Preamble field and Start of Frame Delimiter (SFD) field. A Preamble field contains multiple repeated bit sequence of "01010101" for filtered 2-FSK and multiple repeated bit sequence of "0111 011101110111 ” for filtered 4FSK. An SFD field contains a 16-bit sequence for filtered 2FSK and a 32-bit sequence for filtered 4-FSK. The value of an SFD bit sequence indicates whether the PHY header and the PHY payload are coded or not.
The second part of a PPDU is PHY header (PHR) and it is different in the PPDU with mode switch and the PPDU without mode switch. The mode switch field indicates whether the PPDU is of mode switch or not. When it is zero, the PPDU is one without mode switch and the whole packet is transmitted at a single data rate and single modulation scheme. When it is one, the PPDU is one with mode switch and contains no PHY payload.

The FCS Type field indicates the length of the Frame Check Sequence (FCS). In the 802.15.4 baseline standard, the maximum packet length is of 127 bytes and the FCS length is of 2 bytes. In order to support IP applications in a better way, IEEE $802.15 .4 \mathrm{~g}$ extends the maximum packet length to 2047 bytes, and, correspondingly, adopts a 4-byte FCS as mandatory while keeping 2-byte FCS as optional. When value of an FCS Type field is zero, the FCS length is 4 bytes, and when it is one the FCS length is 2 bytes.

The data whitening (DW) field indicates whether data whitening mechanism is applied. Data whitening is optional in MR-FSK PHY.

4.2.5. Channel Coding. Forward error correction (FEC) channel coding is optional and determined by the SFD value in MR-FSK PHY. It can be either systematic or nonsystematic encoder with a 1/2-rate and constraint length of four. Interleaving of code-bit must be implemented when the convolution code is nonsystematic; however, it is optional when the code is systematic.

4.2.6. Receiver Sensitivity. Receiver sensitivity is the lowest input power for which the PER requirements are met. The 802.15.4 baseline standard requires a PER $<1 \%$ when one measures receiver sensitivity; however, considering the more challenging environment of neighborhood area network and much longer packet length, IEEE 802.15.4g requires a PER < $10 \%$ when receiver sensitivity is measured. Other measurement conditions include a PSDU length of 250 bytes and a 


\begin{tabular}{|c|c|c|c|c|c|c|c|}
\hline Variable & $16 / 32$ & 1 & 2 & 1 & 1 & 11 & Variable \\
\hline Preamble & $\begin{array}{l}\text { Start of } \\
\text { frame } \\
\text { delimiter } \\
\text { (SFD) }\end{array}$ & $\begin{array}{c}\text { Mode } \\
\text { switch }\end{array}$ & $\begin{array}{c}\text { Rese- } \\
\text { rved }\end{array}$ & $\begin{array}{c}\text { FCS } \\
\text { type }\end{array}$ & $\begin{array}{c}\text { Data } \\
\text { whitening }\end{array}$ & $\begin{array}{c}\text { Frame } \\
\text { length }\end{array}$ & $\begin{array}{c}\text { PHY } \\
\text { payload }\end{array}$ \\
\hline \multicolumn{3}{c|}{ PHnchronization } \\
header (SHR)
\end{tabular}

(a) Normal PPDU format of MR-FSK PHY

\begin{tabular}{|c|c|c|c|c|c|c|c|}
\hline Variable & $16 / 32$ & 1 & 2 & 1 & 7 & 4 & 1 \\
\hline Preamble & $\begin{array}{l}\text { Start of } \\
\text { frame } \\
\text { delimiter } \\
\text { (SFD) }\end{array}$ & $\begin{array}{l}\text { Mode } \\
\text { switch }\end{array}$ & $\begin{array}{l}\text { Mode } \\
\text { switch } \\
\text { parame- } \\
\text { ter } \\
\text { entry }\end{array}$ & $\begin{array}{l}\text { New } \\
\text { mode } \\
\text { FEC }\end{array}$ & $\begin{array}{l}\text { New } \\
\text { mode }\end{array}$ & $\begin{array}{c}\text { Checks- } \\
\text { um }\end{array}$ & $\begin{array}{c}\text { Parity } \\
\text { check }\end{array}$ \\
\hline \multicolumn{1}{|c|}{ PHY header (PHR) } \\
Synchronization \\
header (SHR)
\end{tabular}

(b) Mode switch PPDU format of MR-FSK PHY

Figure 2: Two PPDU formats of MR-FSK PHY.

data rate of $50 \mathrm{kbps}$ or higher. Equation (1) is used to calculate the required receiver sensitivity of a device employing MRFSK PHY.

$$
S=S_{0}+10 \log 10\left(\frac{R}{R_{0}}\right)(\mathrm{dBm}),
$$

where $S_{0}$ is -91 without FEC and -97 with FEC, $R_{0}$ is $50 \mathrm{kbps}$, and $R$ is the bit rate in kbps. For data rate of $200 \mathrm{kbps}$, the receiver sensitivity is $-85 \mathrm{dBm}$ without channel coding, while it can be improved to $-91 \mathrm{dBm}$ when channel coding is used.

The practical value of receiver sensitivity in this implementation is around $-100 \mathrm{dBm}$, depending on the specific data rate mode. This is a value produced from repeated testing in the laboratory and field.

\subsection{MAC Layer}

4.3.1. IE Mechanism. IE mechanism has been used by many IEEE standards such as IEEE 802.11 and 802.16, and so forth. It facilitates information exchange at MAC layer between devices with great flexibility brought by modular concepts and designs. A standard with IE mechanism may develop new MAC functions by easily introducing new IEs. IEEE $802.15 .4 \mathrm{e}$ adopted this mechanism to 802.15.4 family by using a new general MAC format as shown in Figure 3. Two types of IEs are employed by IEEE 802.15.4e, that is, header IEs and payload IEs. The former is part of the MAC header (MHR) and is used to process the frame itself, while the latter is a part of MAC payload dealing with next higher layer or service access point (SAP). IEEE 802.15.4g defined a SUN
Device Capabilities IE for exchanging PHY capabilities of data rates, modulation scheme, operating frequencies, and so forth, among SUN devices.

4.3.2. Low-Energy Superframe. To meet low-energy consumption requirement of applications such as SUN, three mechanisms have been defined in $802.15 .4 \mathrm{e}$, that is, lowenergy (LE) superframe, coordinated sampled listening (CSL), and receiver initiated transmission (RIT). The lowenergy (LE) mechanisms are not specific to any particular application domain; they are generic for applications that trade low latency for low-energy consumption. The LE protocol allows devices to operate down to a fraction of $1 \%$ duty cycles while presenting an "always-on" illusion.

Low-energy superframe saves energy consumption of a SUN device by turning off most of or all of the periodic beacon transmissions and by extending inactive periods. When all beacon transmissions are turned off, a coordinator is still capable of transmitting beacons when required to do so.

Figure 4 shows comparison between the legacy IEEE 802.15.4 superframe and the IEEE 802.15.4e LE superframe. A coordinator of the former transmits one beacon every beacon interval (BI), while, for the latter, it transmits a beacon every $N$ BIs, where $N$ can be specified when the network is set up. Moreover, the inactive period is extended by removing the contention-free period (CFP); therefore a SUN device can "sleep" longer to save more energy. In active periods, all SUN devices are awake and monitor data frames transmissions. When a data frame transmission is directed to a SUN device, 


\begin{tabular}{|c|c|c|c|c|c|c|c|c|c|c|}
\hline Octet: $1 / 2$ & $0 / 1$ & $0 / 2$ & $\begin{array}{c}0 / 1 / 2 / \\
8\end{array}$ & $0 / 2$ & $\begin{array}{c}0 / 1 / 2 / \\
8\end{array}$ & $\begin{array}{c}0 / 1 / 5 / 6 / \\
10 / 14\end{array}$ & \multicolumn{2}{|c|}{ Variable } & Variable & 2 \\
\hline Frame & Sequence & $\begin{array}{l}\text { Dest. PAN } \\
\text { indentifier }\end{array}$ & $\begin{array}{l}\text { Dest. } \\
\text { address }\end{array}$ & $\begin{array}{l}\text { Source } \\
\text { PAN } \\
\text { indentifier }\end{array}$ & $\begin{array}{l}\text { Source } \\
\text { address }\end{array}$ & \multirow{2}{*}{$\begin{array}{l}\text { Auxiliary } \\
\text { security } \\
\text { header }\end{array}$} & \multicolumn{2}{|c|}{$\begin{array}{l}\text { Information } \\
\text { element }\end{array}$} & Frame & $\mathrm{E} C \mathrm{~S}$ \\
\hline & & \multicolumn{4}{|c|}{ Addressing fields } & & $\begin{array}{l}\text { Header } \\
\text { IEs }\end{array}$ & $\begin{array}{l}\text { Payload } \\
\text { IEs }\end{array}$ & & \\
\hline \multicolumn{8}{|c|}{ MAC header (MHR) } & \multicolumn{2}{|c|}{ MAC payload } & MFR \\
\hline
\end{tabular}

FIgURE 3: General MAC frame format of IEEE 802.15.4e.

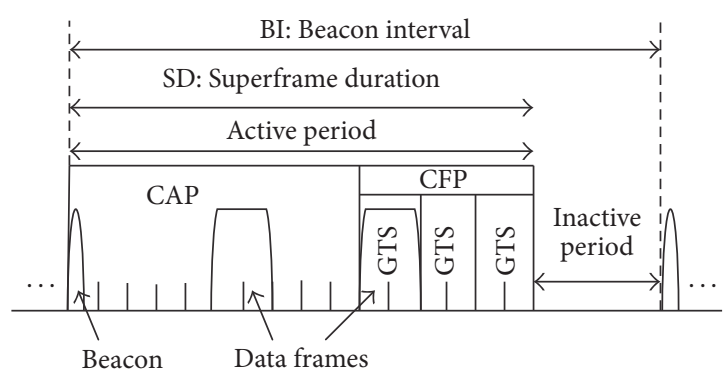

(a) Superframe in IEEE 802.15.4 MAC

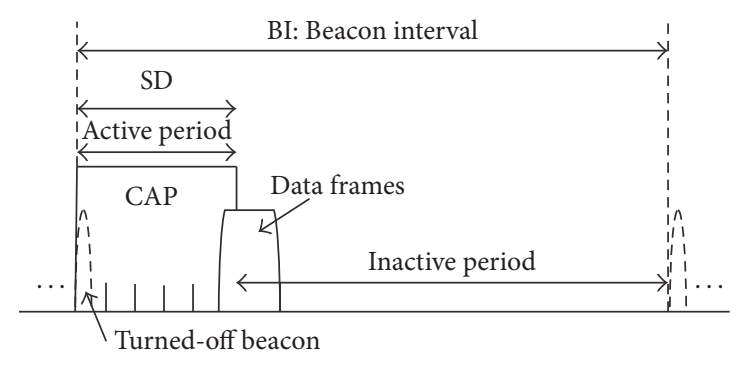

(b) Low-energy superframe

FIGURE 4: Comparison of legacy IEEE 802.15.4 superframe and IEEE 802.15.4e LE superframe structure.

it enables its receiver even during the inactive period, while other SUN devices switch to an inactive state. Data frame transmission shall begin within an active period and be completed before the next active period.

4.3.3. $E B$ and EBR. The 802.15.4e enhanced beacon (EB) is extension of beacons of the baseline standard. An EB is constructed by specific IEs for targeted applications. The EB enables the application related information from higher layers to be periodically broadcast.

The 802.15 .4 e enhanced beacon request (EBR) is extension of beacon request command frame of the baseline standard. It specifies contents of a number of response filter IEs, by which only the neighbors meeting the filtering conditions are requested to response or only the wanted information is requested to be included in the response EBs. By achieving this, an 802.15.4e device may exchange selected MAC or PHY capability information with selected neighbors. This is beneficial for devices to establish communication with neighbor devices with specific MAC or PHY capabilities.

\section{Prototype Implementation}

Figure 5 shows a developed prototype device of a SUN based on IEEE 802.15.4g and 802.15.4e standards [8]. Parameters of the device are listed in Table 2. The operation band is the Japanese band of $920-928 \mathrm{MHz}$. The transmission power is $20 \mathrm{~mW}$ and is expandable to $250 \mathrm{~mW}$, which is the allowed maximum transmission power in this operation band for license-exempt devices in Japan. The modulation scheme is 2GFSK so the implementation cost could be relatively low. The operation data rates are $50 \mathrm{kbps}, 100 \mathrm{kbps}$, and $200 \mathrm{kbps}$, which can be configured in a flexible manner. It supports data packet with size up to 2047 octets. Using 2047 bytes packets enables most reading and applications to work with relatively long packet sizes without requiring packet fragmentation. Adaptive forward error correction (FEC) using a systematic code is implemented so that data transmission in a relatively harsh environment can be supported. At the MAC layer, both beacon and nonbeacon modes are supported and the beacon interval, active period, and inactive period can be configured. The SUN prototype device can be powered by three AA batteries or by AC power. The size of the end product is in the order of several-centimeters-cube. During the active period, the consumption current is $50 \mathrm{~mA}$ and in the inactive sleep state the consumption current is $2 \mathrm{~mA}$. The interfaces of the prototype device include RS-232C, RS-485, U-LINE, and are expandable to others. The prototype is capable of multihop transmissions with the support of both tree and mesh topology. The developed prototype SUN device meets spectrum mask requirement of license-exempt operation in 920-928 MHz band in Japan.

\section{Field Trial Test Scenarios}

By employing the implementation described in Section 5, field trial tests were conducted in outdoor environments. The outdoor test scenarios are as follows: 
TABLE 2: Parameters of the developed SUN devices.

\begin{tabular}{lc}
\hline Size & $85 \mathrm{~mm} 70 \mathrm{~mm} 35 \mathrm{~mm}$ (without antenna) \\
Weight & $165 \mathrm{~g}$ \\
Frequency & $920-928 \mathrm{MHz}$ \\
Tx power & $20 \mathrm{~mW}$ (expandable to $250 \mathrm{~mW})$ \\
Modulation scheme & $2 \mathrm{GFSK}$ \\
Data rate & $50 \mathrm{kbps} ; 100 \mathrm{kbps} ; 200 \mathrm{kbps}$ \\
Maximum data length & 2047 Octet \\
Communication range & $500 \mathrm{~m}$ \\
Power source & AA-battery3; AC \\
Consumption current & $50 \mathrm{~mA}$ for active; $2 \mathrm{~mA}$ for sleep \\
Interface & RS-232C; RS-485; U-LINE, and others (expandable) \\
\hline
\end{tabular}

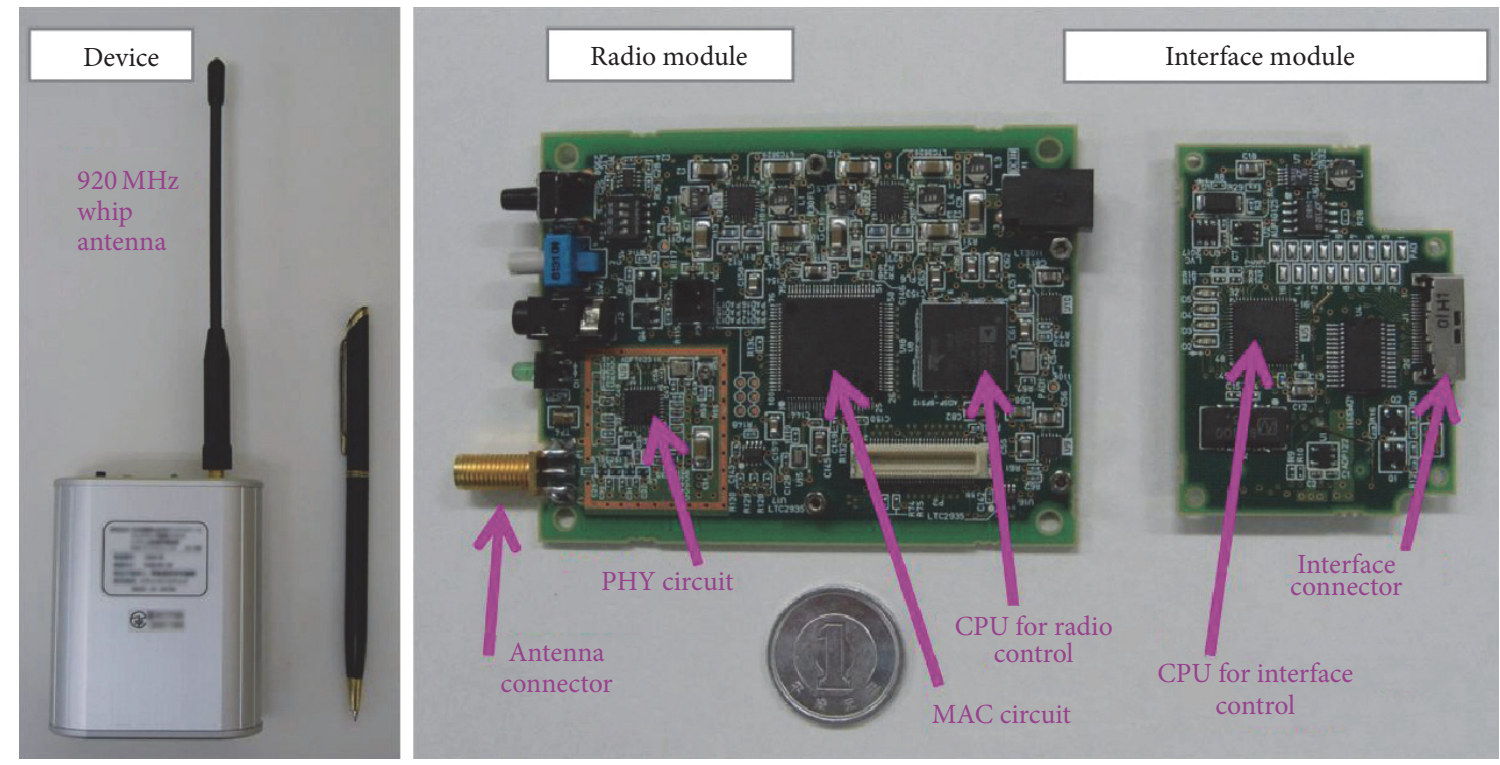

FIGURE 5: Photo of a developed SUN prototype device.

(i) Point-to-point test in line-of-sight (LOS) to determine achievable communication range.

(ii) Star topology test in non-line-of-sight (NLOS) to determine achievable communication range.

(iii) Multiple-node network test along typical building corridor.

(iv) Multiple-node network test across different buildings.

The tested data rate modes were 50,100, and $200 \mathrm{kbps}$. The transmission power was set to $20 \mathrm{~mW}$.

6.1. Line-of-Sight Communication Range Tests. This test was located in East Coast Park Singapore along a straight road and parallel to the coastline. The reason to select this location was to enable a line-of-sight communication between the devices. A data collector (DC) and a node were deployed from each other in line-of-sight in several distances. Both devices were placed 1 meter above the ground. The deployment scenario is shown in Figure 6(a), where $D_{\text {DC-N }}$ is the distance between the DC to the node(s). The packet length was set to 250 octets with transmission interval of $5 \mathrm{~s}$. Data collection was conducted for data rate modes $50 \mathrm{kbps}, 100 \mathrm{kbps}$, and $200 \mathrm{kbps}$. For each data rate mode, several sample points for distance $D_{\mathrm{DC}-\mathrm{N}}$ were taken and communication was established between the DC and the node. Next, data was collected into a laptop with a data logger and processing software. Based on the data, the achievable communication range, above which the $\mathrm{DC}$ was not able to receive any packets, was determined.

6.2. Non-Line-of-Sight Communication Range Tests. This test was setup at Casa Clementi Housing Estate in Singapore. The housing estate consists of multiple blocks as high as 20 storeys. This test site provides a typical housing estate in Singapore with typical building structures and environment. The deployment scenario is shown in Figure 6(b), where a DC was deployed at the 11th and 20th storey of block 419, while two nodes were deployed at the ground floor void deck in block 411 (node N1) and at open space near block 410 (node 


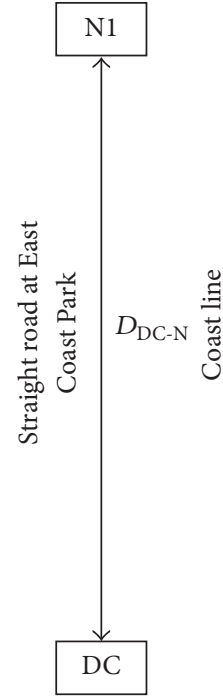

(a)

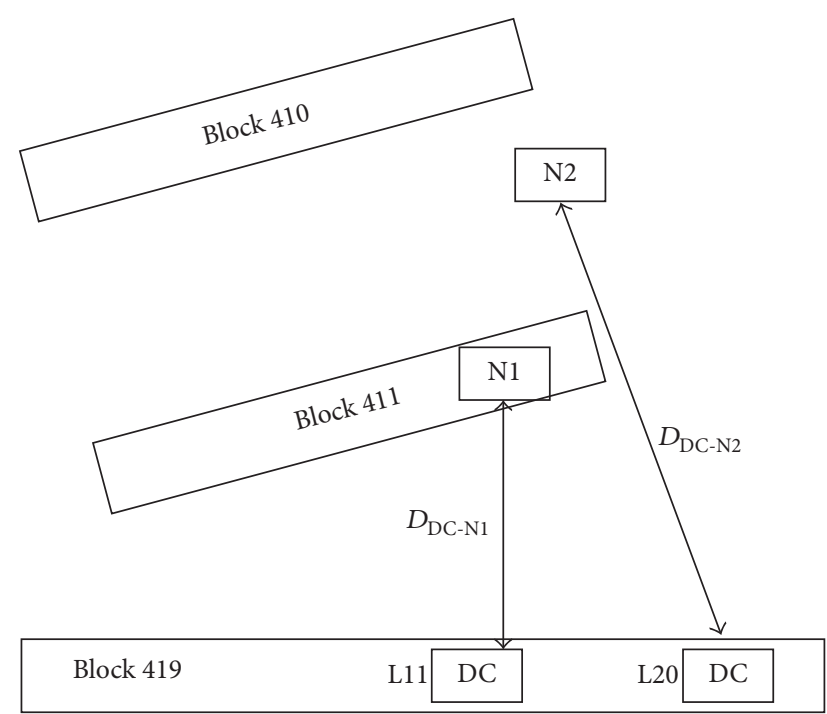

(b)

Figure 6: Deployment scenario for (a) point-to-point LOS test at East Coast Park. (b) Star topology point-to-point NLOS test at housing estate environment. DC: data collector. N: node. L11: 11th storey. L20: 20th storey.

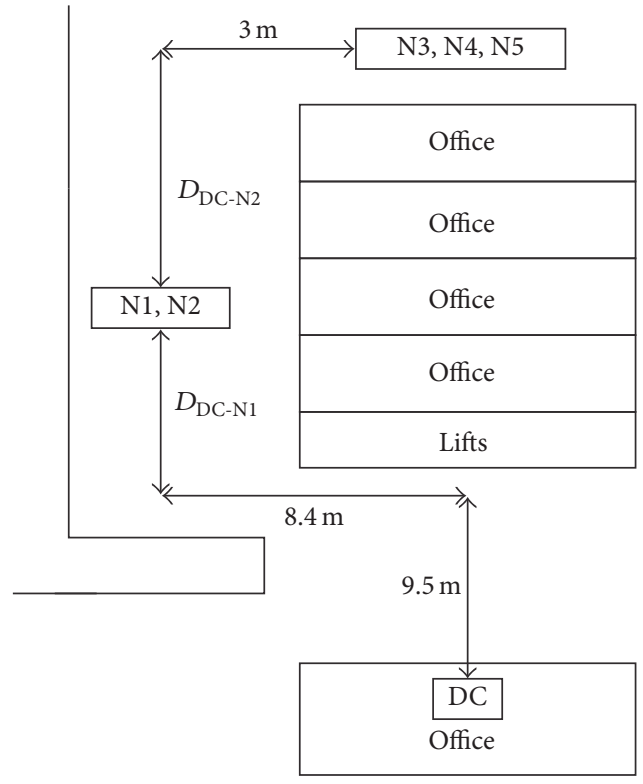

(a)

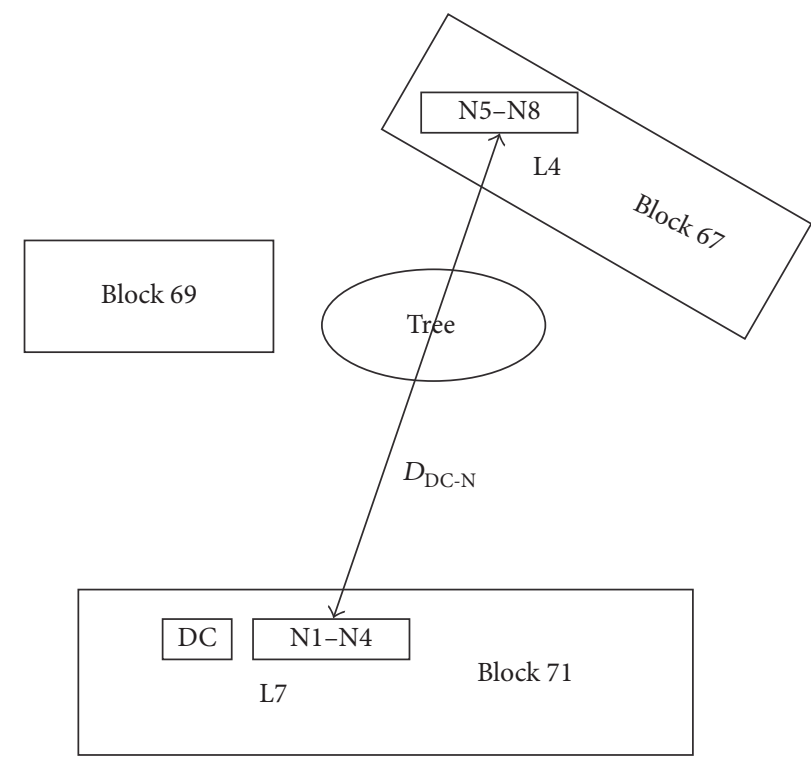

(b)

FIGURE 7: Deployment Scenario for (a) tree topology network test along corridor of office building with devices DC-N1-N3 \& N4 and DCN2N5. (b) Interbuilding line topology network test at office building with devices N1-N5-N2-N6-N3-N7-N4-N8. DC: data collector. N: node.

$\mathrm{N} 2)$, respectively. The heights of the 11th and 20th storeys were $35 \mathrm{~m}$ and $65 \mathrm{~m}$ above the ground. Here, $D_{\mathrm{DC}-\mathrm{N} 1}$ was $75 \mathrm{~m}$ and $D_{\text {DC-N2 }}$ was $103 \mathrm{~m}$.

For each data rate mode, two sample points for the location of DC were taken and the network was established.

6.3. Tree Topology Network along Corridor. This test was setup at Ayer Rajah Industrial Estate in Singapore. This area is filled with multiple-storey buildings for office and factory usage. The environment is a typical industrial estate in Singapore. The illustration of the deployment scenario can be found in Figure 7(a). The network was a tree topology with where DC is connected to $\mathrm{N} 1$ and $\mathrm{N} 2$, while $\mathrm{N} 1$ was connected to $\mathrm{N} 3$ and $\mathrm{N} 4$ and and N2 to N5. This formed a two-path tree topology network. For the $100 \mathrm{kbps}$ tests, $D_{\mathrm{DC}-\mathrm{N} 1}$ and $D_{\mathrm{DC}-\mathrm{N} 2}$ were both $45 \mathrm{~m}$, while for the 
TABLE 3: Results for LOS communication range tests. Reference diagram: Figure 6(a).

\begin{tabular}{|c|c|c|c|}
\hline Performance metrics & & Value & \\
\hline Data rate mode & & $50 \mathrm{kbps}$ & \\
\hline Packets transmitted & 1033 & 1089 & 1045 \\
\hline Packets received & 1020 & 1084 & 1019 \\
\hline Error packets & 1 & 1 & 135 \\
\hline Packet error rate (PER) & $9.8 \times 10^{-4}$ & $9.2 \times 10^{-4}$ & $1.3 \times 10^{-1}$ \\
\hline$D_{\mathrm{DC}-\mathrm{N}}(\mathrm{m})$ & 160 & 300 & 390 \\
\hline Data rate mode & & $100 \mathrm{kbps}$ & \\
\hline Packets transmitted & 1051 & 1097 & 1089 \\
\hline Packets received & 1042 & 1083 & 1012 \\
\hline Error packets & 6 & 30 & 91 \\
\hline Packet error rate (PER) & $5.8 \times 10^{-3}$ & $2.8 \times 10^{-2}$ & $9.0 \times 10^{-2}$ \\
\hline$D_{\mathrm{DC}-\mathrm{N}}(\mathrm{m})$ & 60 & 150 & 240 \\
\hline Data rate mode & & $200 \mathrm{kbps}$ & \\
\hline Packets transmitted & 1108 & 1030 & 1031 \\
\hline Packets received & 1038 & 1013 & 1015 \\
\hline Error packets & 200 & 23 & 63 \\
\hline Packet error rate (PER) & $1.9 \times 10^{-1}$ & $2.2 \times 10^{-2}$ & $6.2 \times 10^{-2}$ \\
\hline$D_{\mathrm{DC}-\mathrm{N}}(\mathrm{m})$ & $160(\mathrm{road})$ & 160 (beach) & 228 (road) \\
\hline
\end{tabular}

$200 \mathrm{kbps}$ tests, $D_{\mathrm{DC}-\mathrm{N} 1}$ and $D_{\mathrm{DC}-\mathrm{N} 2}$ were $16 \mathrm{~m}$ and $30 \mathrm{~m}$, respectively.

6.4. Interbuilding Line Topology Network. This test was setup at Ayer Rajah Industrial Estate in Singapore, across two building blocks 67 (level 4) and 71 (level 7), as shown in Figure 7(b). The deployment scenario was a line network topology with the following connectivity: N1-N5-N2-N6-N3N7-N4-N8. There was obstruction in between the blocks by trees. The DC was located nearby N1 to N4 in block 71. Here, $D_{\text {DC-N }}$ was approximately $100 \mathrm{~m}$.

\section{Performance Evaluation}

Deploying the test scenario described in Section 6, data collection was conducted and analyzed. The following presents the results of the measurement activities.

An important performance metric is the packet error rate (PER), expressed as the fraction of error packets to the total received packets in the receiver. It is worth noting that the required PER as per [6] is $10 \%$. This value will be used as the reference in evaluating the performance throughout this study.

7.1. Performance in LOS Communication Range Tests. Table 3 presents the measured data in the LOS communication range tests. The data consists of data rate modes $50 \mathrm{kbps}, 100 \mathrm{kbps}$, and $200 \mathrm{kbps}$.

A general observation was that, although [6] specified PER $=10 \%$ as the critical PER rate, in the field tests, it is difficult to obtain the distance whereby the PER becomes $10 \%$. Instead, the common occurrence was that, above a certain distance (i.e., the achievable communication range), PER quickly increased from $0 \%$ significantly. From the tests, the achievable communication ranges for $50 \mathrm{kbps}, 100 \mathrm{kbps}$, and $200 \mathrm{kbps}$ were $450 \mathrm{~m}, 240 \mathrm{~m}$, and $260 \mathrm{~m}$, respectively. This is due to the fact that at a fixed power of $20 \mathrm{~mW}$, higher data rates result in less energy per transmitted bit, thus degrading the performance. From Table 3, it was understood that PER increased as $D_{\mathrm{DC}-\mathrm{N}}$ increased. This can be explained that more packets are received in error with the longer propagation path due to reduction power density over distance. Theoretically, a $20 \mathrm{~mW}$ device should be able to achieve the range up to $1 \mathrm{~km}$. In this test, we explain that the shorter achievable range in LOS environment is due to the fact that it is not a perfect LOS. There are still obstructions and reflectors impeding the achievable range of the device.

For data rate mode $200 \mathrm{kbps}$, two test scenarios were employed, one along the road and one at the beach, both locations are LOS environment. PER for the link along the road was $19 \%$ while that at the beach was $2.2 \%$. Considerable factors are the larger volume of signal noise created by vehicles on the road being more significant that the human traffic at the beach.

7.2. Performance in NLOS Communication Range Tests. Table 4 presents the measured data in the NLOS communication range tests. All parameter settings of the DC and node were the same. The location was setup at a typical housing estate in Singapore with flats up to 20 storeys. The DC was located placed at level 11 and level 20, with N1 placed at ground floor across an adjacent building. Additionally, N2 was placed connected to N1 to form a star topology network. The materials of construction for the buildings are cement and bricks.

The data was collected corresponding to different locations of the DC at different storeys. It was found that, at 
TABLE 4: Results for NLOS communication range tests. Reference diagram: Figure 6(b). In the same row, the upper value denotes DC location on the 11th storey L11 and the lower value denotes DC location on the 20th storey L20.

\begin{tabular}{|c|c|c|c|}
\hline Performance metrics & & Values & \\
\hline Data rate mode & & $50 \mathrm{kbps}$ & \\
\hline Node & N1 & & $\mathrm{N} 2$ \\
\hline Distance, DC-N1 \& DC-N2 (m) & 75 & & 103 \\
\hline \multirow{2}{*}{ Packets received } & L11: 1069 & & L11: 1089 \\
\hline & L20: 1009 & & L20: 1012 \\
\hline \multirow{2}{*}{ Error packets } & L11: 3 & & L11: 0 \\
\hline & L20: 172 & & L20:2 \\
\hline \multirow{2}{*}{ Packet error rate (PER) } & L11: $2.8 \times 10^{-3}$ & & L11: 0 \\
\hline & L20: $1.7 \times 10^{-1}$ & & L20: $2.0 \times 10^{-3}$ \\
\hline Data rate mode & & $100 \mathrm{kbps}$ & \\
\hline Node & $\mathrm{N} 1$ & & $\mathrm{~N} 2$ \\
\hline Distance, DC-N1 \& DC-N2 (m) & 75 & & 103 \\
\hline \multirow{2}{*}{ Packets received } & L11: 1050 & & L11: 1146 \\
\hline & L20: 1024 & & L20: 433 \\
\hline \multirow{2}{*}{ Error packets } & L11: 0 & & L11: 154 \\
\hline & L20: 204 & & L20: 110 \\
\hline \multirow{2}{*}{ Packet error rate (PER) } & L11: 0 & & L11: $1.3 \times 10^{-1}$ \\
\hline & L20: $2.0 \times 10^{-1}$ & & L20: $2.5 \times 10^{-1}$ \\
\hline Data rate mode & & $200 \mathrm{kbps}$ & \\
\hline Node & N1 & & $\mathrm{N} 2$ \\
\hline Distance, DC-N1 \& DC-N2 (m) & 75 & & 103 \\
\hline \multirow{2}{*}{ Packets received } & L11: 1924 & & L11: 1095 \\
\hline & - & & - \\
\hline \multirow{2}{*}{ Error packets } & L11: 48 & & L11: 33 \\
\hline & - & & - \\
\hline \multirow{2}{*}{ Packet error rate (PER) } & L11: $2.5 \times 10^{-2}$ & & L11: $3.0 \times 10^{-2}$ \\
\hline & - & & - \\
\hline
\end{tabular}

level 11, for all data rate modes, the measured PER was at least two digits better than the required PER of $10 \%$. On the other hand, when the DC was moved to level 20, the PER becomes higher than $10 \%$ for the lower data rate modes $50 \mathrm{kbps}$ and $100 \mathrm{kbps}$. For the $200 \mathrm{kbps}$ mode, connection was not able to be established. This indicated that for a 20 storey building, one relay device (i.e., two-hop network) at the 11th storey is sufficient to cover the network of the entire building vertically. Another observation was that the PER at N1 and $\mathrm{N} 2$ are not significantly different from each other. The reason is due to the transmission path of DC to N1 and N1 to N2 were obstructed with almost similar numbers of walls.

Other findings showed that PER increases as the data rate, number of concrete walls in between, and distance increase. In general, a one-hop $20 \mathrm{~mW}$ network is capable of covering up to 11th storey vertically and across one block horizontally.

7.3. Performance in Tree Topology Network Tests along Corridor. Table 5 presents the measured data of the tree topology network in along a corridor. Data rate modes $100 \mathrm{kbps}$ and $200 \mathrm{kbps}$ were used for this test. The test venue was a concrete building for office and industrial establishments. The network deployment scenario was tree topology where the DC was connected to two second-tier nodes, N1 and N2, while the third-tier was N3, N4, and N5. The distances among different nodes were described in Figure 7(a).

Based on the collected data, it was found that the network for both data rate modes $100 \mathrm{kbps}$ and $200 \mathrm{kbps}$ had no problem being deployed in the test site. Performance of almost no error packets can be achieved with such a setup. This indicates that for a typical office/industrial building, the network capability is sufficient to support a full range coverage.

7.4. Performance in Line Topology Network Test for Interbuilding Communications. Table 6 presents the test data of the interbuilding line topology network. In this setup, the signal was bounced to and from across two buildings. The DC, $\mathrm{N} 1$ through $\mathrm{N} 4$ were placed in the same building, while N5 through N8 were placed at another building 100 meters apart. The buildings were concrete office/industrial facilities with tree obstructing in between. The illustration was given in Figure $7(\mathrm{~b})$. There was a total of eight tiers of hierarchical structure in the line topology network. 
TABLE 5: Results for tree topology network tests along corridor. Reference diagram: Figure 7(a).

\begin{tabular}{|c|c|c|c|c|c|}
\hline \multirow{2}{*}{$\begin{array}{l}\text { Performance metrics } \\
\text { Node }\end{array}$} & \multicolumn{5}{|c|}{ Values } \\
\hline & N1 & $\mathrm{N} 2$ & N3 & N4 & N5 \\
\hline Data rate mode & & & $100 \mathrm{kbps}$ & & \\
\hline Packets transmitted & 110 & 108 & 104 & 163 & 102 \\
\hline Packets received & 98 & 101 & 103 & 98 & 88 \\
\hline Error packets & 2 & 1 & 0 & 1 & 1 \\
\hline $\operatorname{PER}\left(\times 10^{-2}\right)$ & 2.0 & 1.0 & 0 & 1.0 & 1.1 \\
\hline Data rate mode & & & $200 \mathrm{kbps}$ & & \\
\hline Packets transmitted & 67 & 67 & 68 & 68 & 67 \\
\hline Packets received & 65 & 63 & 63 & 68 & 63 \\
\hline Error packets & 0 & 0 & 0 & 0 & 0 \\
\hline $\operatorname{PER}\left(\times 10^{-2}\right)$ & 0 & 0 & 0 & 0 & 0 \\
\hline
\end{tabular}

TABLE 6: Results for line topology network test for interbuilding communications. Reference diagram: Figure 7(b).

\begin{tabular}{|c|c|c|c|c|c|c|c|c|}
\hline \multirow{2}{*}{$\begin{array}{l}\text { Metrics } \\
\text { Node }\end{array}$} & \multicolumn{8}{|c|}{ Values } \\
\hline & N1 & $\mathrm{N} 2$ & N3 & $\mathrm{N} 4$ & N5 & N6 & N7 & N8 \\
\hline Data rate mode & \multicolumn{8}{|c|}{$100 \mathrm{kbps}$} \\
\hline Packets transmitted & 80 & 80 & 80 & 80 & 80 & 81 & 80 & 80 \\
\hline Packets received & 69 & 80 & 79 & 78 & 77 & 79 & 77 & 79 \\
\hline Error packets & 0 & 1 & 2 & 1 & 0 & 0 & 1 & 1 \\
\hline $\operatorname{PER}\left(\times 10^{-2}\right)$ & 0 & 1.3 & 2.5 & 1.3 & 0 & 0 & 1.3 & 1.3 \\
\hline Data rate mode & \multicolumn{8}{|c|}{$200 \mathrm{kbps}$} \\
\hline Packets transmitted & 87 & 87 & 86 & 87 & 86 & 87 & 86 & 87 \\
\hline Packets received & 85 & 85 & 83 & 84 & 80 & 85 & 83 & 85 \\
\hline Error packets & 4 & 3 & 3 & 3 & 7 & 6 & 7 & 5 \\
\hline $\operatorname{PER}\left(\times 10^{-2}\right)$ & 4.7 & 3.5 & 3.6 & 3.6 & 8.6 & 7.1 & 8.4 & 5.8 \\
\hline
\end{tabular}

The measured data showed only 1 or 2 error from the total received packets. This indicated that the PER was well below the required PER in [6].

\section{Results Analysis}

This section provides analysis based on the data gathered in the test described in Section 7 and how the test results correspond to the requirements in the use cases listed in Section 3.

Use case 1 requires the support of a typical range between nodes in an open area with LOS environment. From the test results in Section 7.1, the achievable ranges for SUN $50 \mathrm{kbps}, 100 \mathrm{kbps}$, and $200 \mathrm{kbps}$ data rate modes were $450 \mathrm{~m}$, $240 \mathrm{~m}$, and $260 \mathrm{~m}$. It was observed that the supportable range is capable of facilitating the use case featuring the connectivity among nodes in an open air. The distance between two buildings in a typical city such as Singapore does not go beyond $450 \mathrm{~m}$. This indicates that the $50 \mathrm{kbps}$ mode is sufficient to connect most buildings. Additionally, for buildings separated in closer proximity within $200 \mathrm{~m}$, higher data rates can be employed to establish a faster utility network.
Use case 2 requires the achievable typical range between nodes in an NLOS environment. Section 7.2 showed that for a typical deployment scenario, such as a residential multistorey building in Singapore, the achievable range for SUN $50 \mathrm{kbps}$, $100 \mathrm{kbps}$, and $200 \mathrm{kbps}$ data rate modes is at least 11th storey, that is, vertically $35 \mathrm{~m}$ above the ground. Beyond the 11th storey, the PER of the system decreases gradually. At the 20th storey, the PER is found to exceed the required performance value of $10 \%$. This indicates that for a 20 -storey building in Singapore, a two-hop network is sufficient to support the utility network, up to $200 \mathrm{kbps}$. This data rate is sufficient to cater the demands for most utility command-and-control features.

Use case 3 highlights the requirement of multihop network establishment in a dense urban environment. Based on the results in Section 7.3, it is evident that the network is capable of enabling the network establishment and communication within an office/industrial premise. The network was deployed along an outdoor corridor, most of which involved NLOS signal propagation among nodes from various corners of the premise. The results revealed that in a typical office/industrial building spanning across approximately $100 \mathrm{~m}$ in length the SUN multihop tree network was capable 
of recording performance meeting the PER requirements of $10 \%$ for both $100 \mathrm{kbps}$ and $200 \mathrm{kbps}$ data rate modes. With reasonable extrapolation, the $50 \mathrm{kbps}$ mode which is relatively more robust should also be able to deliver the same, if not better performance.

Use case 4 outlines the system requirements for communication networks with extended range to cater scenarios such as expansion to outer regions of the core network area. Results from Section 7.4 showed that the SUN network is able to establish and maintain an eight-hop network across buildings, penetrating through obstruction such as trees. The network is able to achieve required PER of $10 \%$ while extending the range up to eight fold. Similarly, the network is able to support the $100 \mathrm{kbps}$ and $200 \mathrm{kbps}$ data rate modes. This indicates that, for a particular region outside a dense area, range extension through increasing the number of hops can be employed to address the traffic demands of that region alone. This feature is especially useful in extending the range from a dense urban area to suburban areas.

All the four use cases can be combined to generically model the typical deployment scenario in an urban city. This field trial has covered most of the anticipated network deployment in such settings. Therefore, the results of this field trial may be extended and reused to analyze performance in similar areas.

\section{Conclusion}

This paper has presented the field trial results of the IEEE 802.15.4/4g/4e SUN in applications suited for outdoor environment. It was concluded that the system is able to meet the requirements set forth by the practical application scenarios. This study has verified that the SUN system can be applied in real world environments to facilitate the communications of applications of similar requirements such as utility networks, machine-to-machine networks, and sensor networks. Future works involve more tests in other environments including indoor locations.

\section{Disclosure}

At the time of publication, Dr. Ming-Tuo Zhou is affiliated with Key Lab of Wireless Sensor Network and Communication, Chinese Academy of Science.

\section{Conflicts of Interest}

The authors declare that they have no conflicts of interest.

\section{Acknowledgments}

The authors wish to thank Infocomm Development Authority (IDA) of Singapore for facilitating the trial program and BioMachines Pte. Ltd. for conducting the tests and producing the evaluation reports.

\section{References}

[1] NIST Special Publication, NIST Framework and Roadmap for Smart Grid Interoperability Standards Release 3.0, National Institute of Standards and Technology, 2014.

[2] C.-S. Sum, L. Lu, M.-T. Zhou, F. Kojima, and H. Harada, "System evaluation of a practical IEEE 802.15.4/4e/4g multi-physical and multi-hop smart utility network," IET Communications, vol. 9, no. 5, pp. 665-673, 2015.

[3] S. Abdul Salam, S. A. Mahmud, G. M. Khan, and H. S. AlRaweshidy, "M2M communication in Smart Grids: implementation scenarios and performance analysis," in Proceedings of the IEEE Wireless Communications and Networking Conference (WCNCW '12), pp. 142-147, IEEE, Paris, France, April 2012.

[4] S. Rajagopal, M. Trayer, N. Nguyen, and K. P. Bhat, "Architecture model choices for a Smart Grid home network," in Proceedings of the 1st IEEE Online Conference on Green Communications (GreenCom '11), pp. 52-57, September 2011.

[5] IEEE, "IEEE standard for information technology-telecommunications and information exchange between systems-local and metropolitan area networks-specific requirements-part 15.4: wireless Medium Access Control (MAC) and Physical Layer (PHY) specifications for low-rate Wireless Personal Area Networks (WPANs)," IEEE Standard 802.15.4-2011, 2011.

[6] IEEE, "Part 15.4: wireless Medium Access Control (MAC) and Physical Layer (PHY) specifications for low-rate Wireless Personal Area Networks (WPANs) - amendment 4: physical layer specifications for low data rate wireless smart metering utility networks," IEEE Standard 802.15.4g-2012, 2012.

[7] "IEEE Std. 802.15.4e-2012, Part 15.4: Wireless Medium Access Control (MAC) and Physical Layer (PHY) Specifications for Low-Rate Wireless Personal Area Networks (WPANs): Amendment to the MAC sub-layer," March 2012.

[8] F. Kojima and H. Harada, "Study on multipath characteristics for IEEE $802.15 .4 \mathrm{~g}$ SUN applications in the frequency band used in Japan," in Proceedings of the 2010 IEEE International Conference on Communications Workshops (ICC '10), pp. 1-5, Cape Town, South Africa, May 2010.

[9] R. Ma, H.-H. Chen, Y.-R. Huang, and W. Meng, "Smart grid communication: its challenges and opportunities," IEEE Transactions on Smart Grid, vol. 4, no. 1, pp. 36-46, 2013.

[10] C.-S. Sum, F. Kojima, and H. Harada, "Performance analysis of a multi-hop IEEE 802.15.4g OFDM system in multi-PHY layer network," in Proceedings of the IEEE 24th Annual International Symposium on Personal, Indoor, and Mobile Radio Communications (PIMRC '13), pp. 1538-1542, London, UK, September 2013.

[11] C.-S. Sum, F. Kojima, and H. Harada, "Energy consumption evaluation for power saving mechanisms in recent IEEE 802.15.4 low-rate wireless personal area networks," in Proceedings of the IEEE International Conference on Communications (ICC '13), pp. 4449-4454, Budapest, Hungary, June 2013.

[12] C.-S. Sum, M. A. Rahman, Z. Lan, F. Kojima, R. Funada, and H. Harada, "Performance analysis of low duty FSK system for Smart Utility Network," in Proceedings of the IEEE Wireless Communications and Networking Conference (WCNC '11), pp. 1568-1573, Cancun, Mexico, March 2011.

[13] C.-S. Sum, F. Kojima, and H. Harada, "Enhanced beaconless synchronization for regulatory domain specific IEEE $802.15 .4 \mathrm{~g}$ smart utility networks," in Proceedings of the IEEE Vehicular Technology Conference (VTC Fall '12), pp. 1-6, Quebec City, Canada, September 2012. 
[14] D. Niyato, L. Xiao, and P. Wang, "Machine-to-machine communications for home energy management system in smart grid," IEEE Communications Magazine, vol. 49, no. 4, pp. 53-59, 2011.

[15] G. Iyer, P. Agrawal, E. Monnerie, and R. S. Cardozo, "Performance analysis of wireless mesh routing protocols for smart utility networks," in Proceedings of the IEEE 2nd International Conference on Smart Grid Communications (SmartGridComm '11), pp. 114-119, IEEE, Brussels, Belgium, October 2011.

[16] P. Muthukumaran, R. de Paz, R. Spinar, and D. Pesch, "MeshMAC: enabling mesh networking over IEEE 802.15.4 through distributed beacon scheduling," in Proceedings of the 1st International Conference (ADHOCNETS '09), pp. 561-575, Ontario, Canada, September 2009.

[17] C.-S. Sum, M. A. Rahman, L. Lu, F. Kojima, and H. Harada, "On communication and interference range of IEEE 802.15.4 g smart utility networks," in Proceedings of the 2012 IEEE Wireless Communications and Networking Conference (WCNC '12), pp. 1169-1174, Paris, France, April 2012.

[18] C.-S. Sum, F. Kojima, and H. Harada, "Coexistence of homogeneous and heterogeneous systems for IEEE $802.15 .4 \mathrm{~g}$ smart utility networks," in Proceedings of the IEEE International Symposium on Dynamic Spectrum Access Networks (DySPAN '11), pp. 510-520, Aachen, Germany, May 2011.

[19] C.-S. Sum, H. Harada, F. Kojima, and L. Lu, "An interference management protocol for multiple physical layers in IEEE 802.15.4g smart utility networks," IEEE Communications Magazine, vol. 51, no. 4, pp. 84-91, 2013.

[20] C.-S. Sum, F. Kojima, and H. Harada, "Performance analysis of a multi-PHY coexistence mechanism for IEEE 802.15.4 g FSK network," in Proceedings of the 2013 IEEE Wireless Communications and Networking Conference (WCNC '13), pp. 41-46, Shanghai, China, April 2013.

[21] J. A. J. De Oliveira, A. F. R. Queiroz, E. R. De Lima, and J. Mertes, "An MR-FSK transceiver compliant to IEEE802.15.4g for smart metering utility applications: FPGA implementation and ASIC resource estimation," in Proceedings of the 7th IEEE Latin-American Conference on Communications (LATINCOM '15), pp. 1-4, November 2015.

[22] M.-K. Oh, J.-Y. Kim, S. Lee, Y. Jeon, and S. Choi, "A fully integrated ieee IEEE 802.15.4g MR-FSK SoC soc for smart utility network applications," IEEE Transactions on Consumer Electronics, vol. 60, no. 4, pp. 580-586, 2014.

[23] D. C. Alves, G. S. Da Silva, E. R. De Lima et al., "Architecture design and implementation of key components of an OFDM transceiver for IEEE 802.15.4 g," in Proceedings of the 2016 IEEE International Symposium on Circuits and Systems (ISCAS '16), pp. 550-553, Montreal, Canada, May 2016.

[24] J. Trefke, J. M. Gonzalez, and M. Uslar, "Smart Grid standardisation management with use cases," in Proceedings of the 2012 IEEE International Energy Conference and Exhibition (ENERGYCON '12), pp. 903-908, Florence, Italy, September 2012.

[25] A. Zaballos, A. Vallejo, and J. M. Selga, "Heterogeneous communication architecture for the smart grid," IEEE Network, vol. 25, no. 5, pp. 30-37, 2011.

[26] Technical Specification for Short Range Devices, IDA TS SRD, Issue 1 Rev 4, Infocomm Development Authority of Singapore, July 2009. 


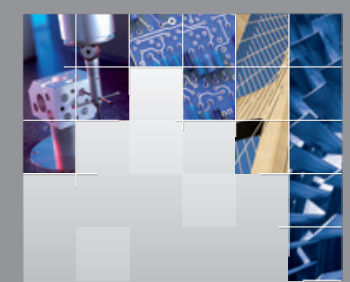

\section{Enfincering}
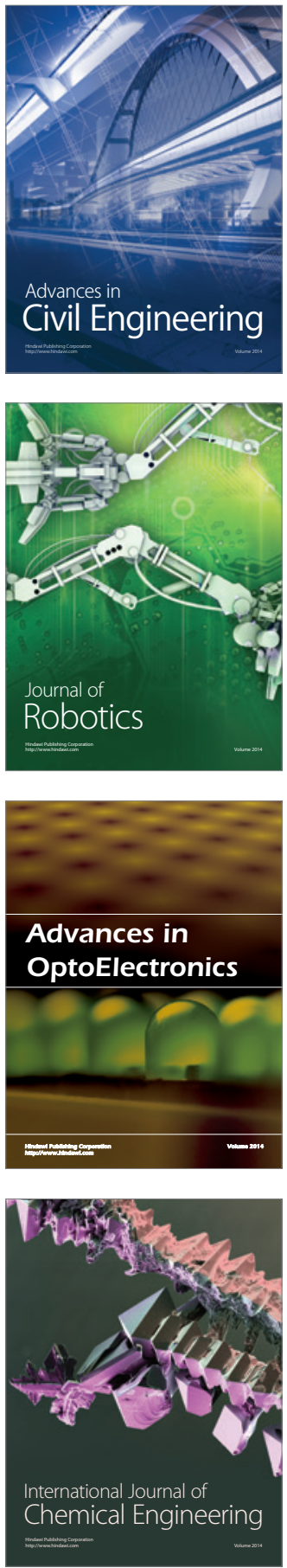

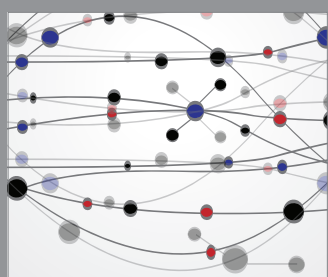

The Scientific World Journal

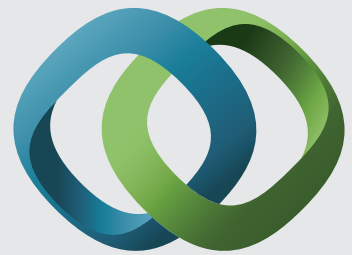

\section{Hindawi}

Submit your manuscripts at

https://www.hindawi.com
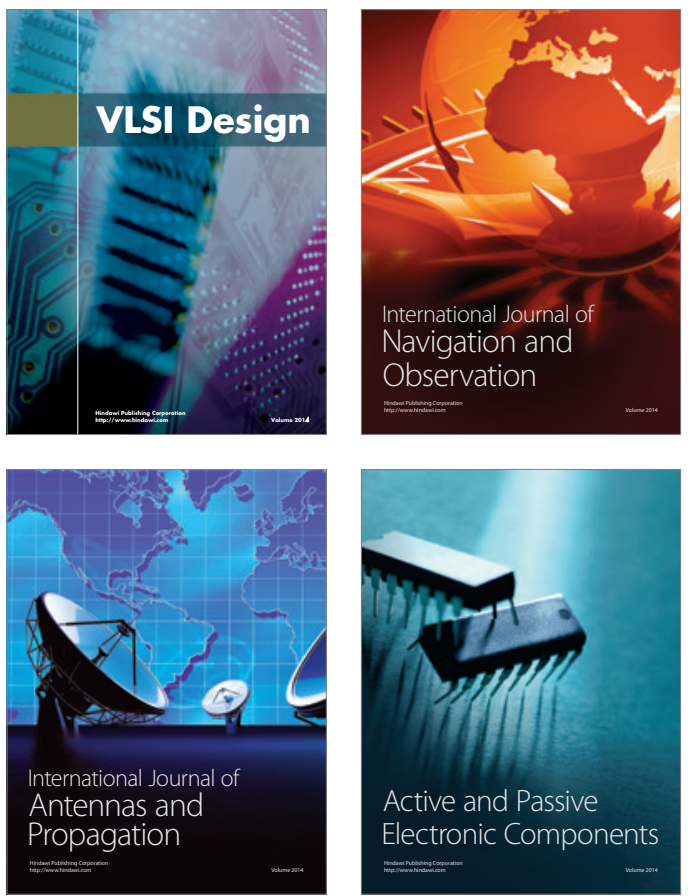
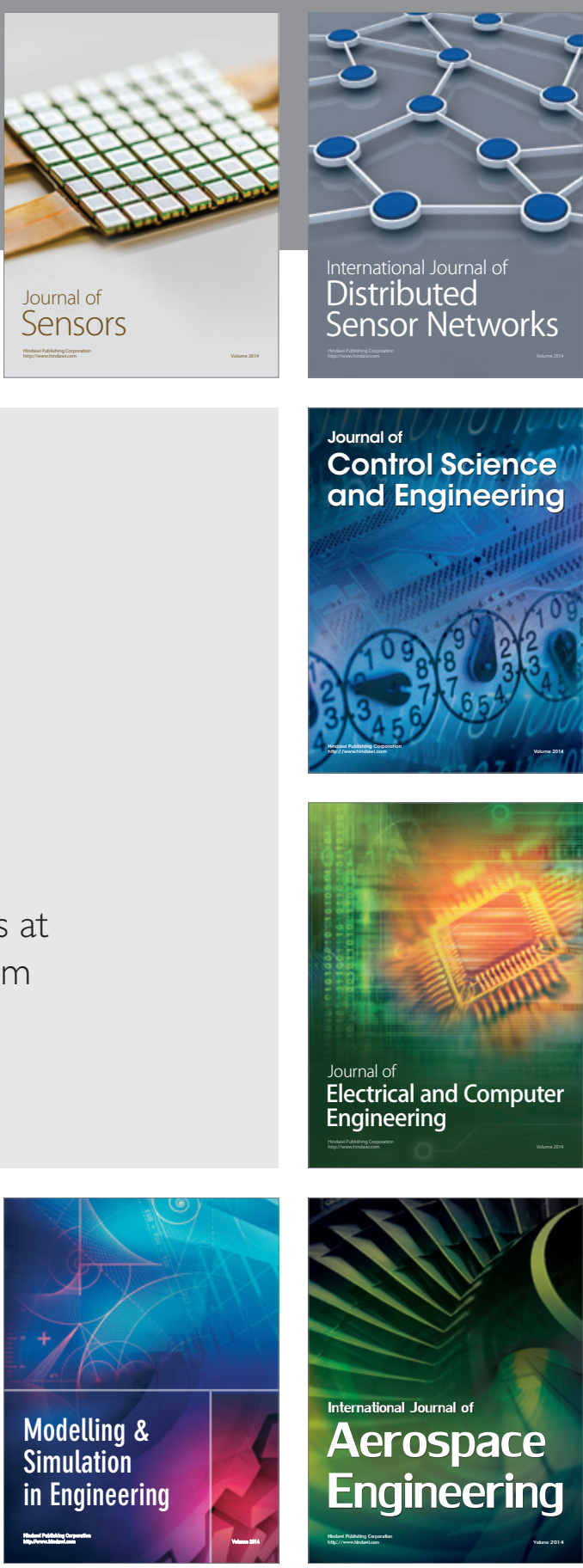

International Journal of

Distributed

Sensor Networks

$-$

Joumal of

Control Science

and Engineering
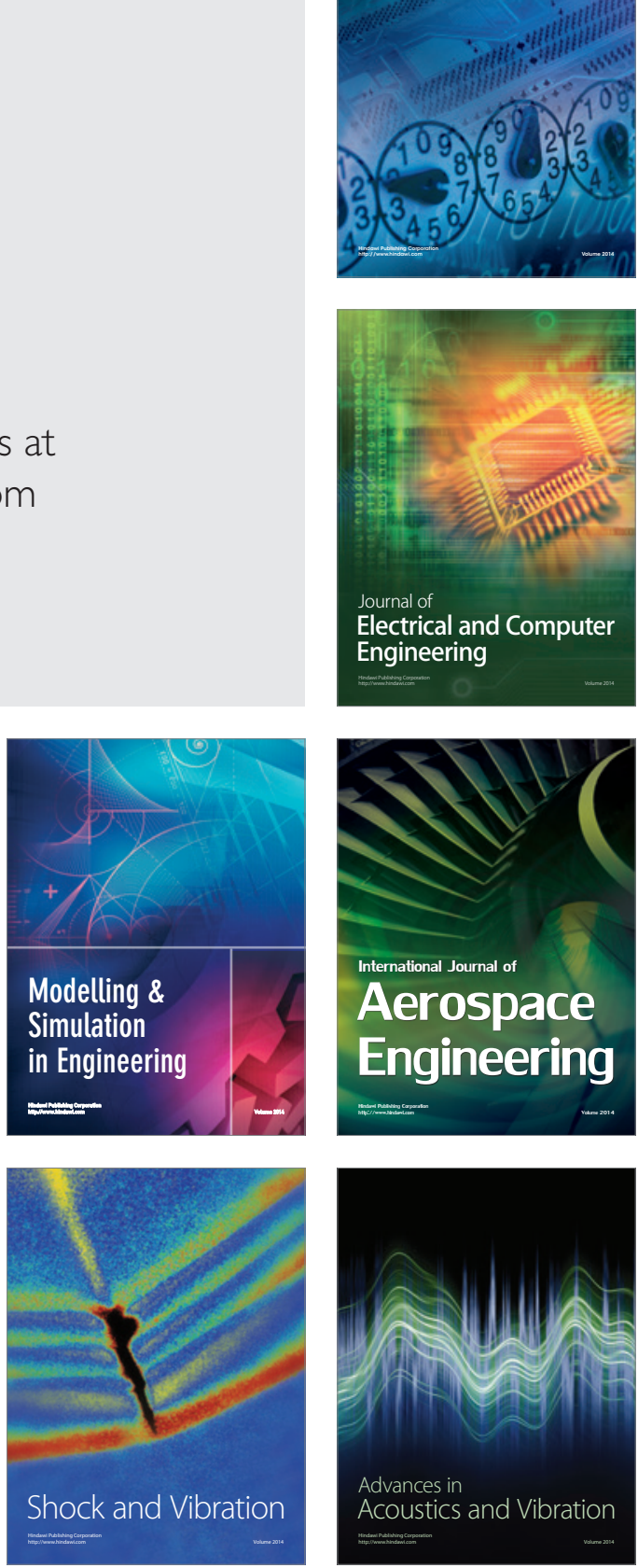